\section{Efeitos da administração de metformina sobre a pressão arterial e o metabolismo glicídico de ratos espontaneamente hipertensos tornados obesos pela injeção neonatal de glutamato monossódico}

\author{
Metformin effects upon blood pressure and glucose metabolism of \\ monossodium glutamate induced-obese spontaneously hypertensive rats
}

Carolina B. N. D. Ferreira', Mário Luís R. Cesaretti', Milton Ginoza', Osvaldo Kohlmann Jr. ${ }^{1}$

\section{RESUMO}

Objetivos: Produzir um modelo experimental de síndrome metabólica (SM) e analisar efeitos da metformina sobre pressão arterial (PA), peso corporal (PC), metabolismo glicídico e conteúdo de gordura epididimal (GE). Método: Os machos SHR receberam $2 \mathrm{mg} / \mathrm{kg} / \mathrm{dia}$ de glutamato monossódico (MSG) até o $11^{\circ}$ dia de vida. Os controles receberam salina. Após 12 semanas, foram separados em dois grupos e tratados com $500 \mathrm{mg} / \mathrm{kg} / \mathrm{dia}$ de metformina ou veículo. Foram acompanhados a PA e o PC dos dois grupos. Ao final do seguimento, realizou-se o teste de tolerância à glicose oral (TTGO) e mediu-se o índice de sensibilidade à insulina. Após sacrifício dos animais, a GE foi pesada. Resultados: A administração de MSG intensificou a resistência insulínica e aumentou o conteúdo de GE, sem, no entanto, alterar a PA. O tratamento com metformina promoveu melhora da sensibilidade insulínica e redução da GE e PA. Conclusões: Observou-se importante papel da resistência hepática à insulina na SM e efeitos cardiovasculares benéficos da melhora na sensibilidade insulínica. Arq Bras Endocrinol Metab. 2009;53(4):409-15.

Descritores

Resistência à insulina; hipertensão arterial; obesidade

\begin{abstract}
Objectives: To make available experimental model for the metabolic syndrome (MS) and verify effects of chronic oral treatment with metformin upon blood pressure (BP), body weight (BW), glucose metabolism, epididimal fat content (EF). Method: Males SHR received monossodium glutamate (MSG, $2 \mathrm{mg} / \mathrm{kg} / \mathrm{day} / \mathrm{sc}$ ) during first 11 days of life. Control animals received saline. After 12 weeks, animals were separated in two groups, treated either with metformin $500 \mathrm{mg} /$ $\mathrm{kg} /$ day or vehicle during 12 weeks. PA and BW were determined. At the end of the follow-up, animals underwent an oral glucose tolerance test (OGTT) and insulin sensitivity index was determined. Upon sacrifice EF was measured. Results: MSG worsened insulin resistance and induced visceral obesity in SHR, without change BP. Treatment with metformin improved glucose metabolism and reduces EF and BP. Conclusions: These observations emphasize the role of hepatic insulin resistance on MS and point out for beneficial cardiovascular effects with improvement in the insulin sensitivity. Arq Bras Endocrinol Metab. 2009;53(4):409-15.
\end{abstract}

Keywords

Insulin resistance; hypertension; obesity
Laboratório de Hipertensão Arterial, Disciplina de Nefrologia, Universidade Federal de São Paulo (Unifesp)

' Disciplina de Nefrologia, Unifesp, São Paulo, SP, Brasil

Correspondência para: Osvaldo Kohlmann Jr. Disciplina de Nefrologia da Unifesp Rua Botucatu, 740 04023-900 - São Paulo, SP, Brasil kohlmann@hrim.com.br

Recebido em 15/Dez/2008 Aceito em 28/Fev/2009 


\section{INTRODUÇÃO}

A síndrome metabólica (SM) caracteriza-se pelo conjunto de fatores de risco para aterosclerose e doença cardiovascular. Essa síndrome tornou-se o protótipo do estilo de vida atual marcado pelo estresse psicossocial, pela mudança de hábitos alimentares e pela diminuição da prática de atividade física. Atualmente, existem várias definições para a SM, mas todas incluem resistência à insulina, hipertensão arterial, obesidade visceral e dislipidemia. Embora não se saiba a causa exata da $\mathrm{SM}$, a resistência à insulina e a adiposidade visceral são consideradas como os principais fatores envolvidos na fisiopatogenia desse conjunto de desordens (1-5). Tais aspectos associados à capacidade do tecido adiposo de produzir angiotensinogênio e à ativação do sistema nervoso simpático e da reabsorção tubular de sódio pela insulina tornam possível a compreensão do desenvolvimento da hipertensão arterial nessa síndrome (6-8).

A SM, porém, é difícil de ser estudada em animais de experimentação. Modelos experimentais, como ratos espontaneamente hipertensos, também são resistentes à insulina, porém não são obesos. Já modelos de ratos obesos, como o Zucker e o induzido pela administração neonatal de glutamato monossódico, são resistentes à insulina, porém não são hipertensos (9). Faz-se necessária a busca de modelo experimental que mimetize as características da SM humana.

Como a resistência à insulina representa papel-chave entre todas as alterações encontradas na SM, o tratamento farmacológico dessa disfunção metabólica pode levar ao controle tanto das anormalidades glicêmicas quanto da elevação dos níveis pressóricos.

A metformina é uma droga amplamente utilizada no tratamento de pacientes portadores de diabetes melito tipo 2 (DM2). Essa droga tem revelado diversos efeitos, como estímulo da atividade quinase dos receptores de insulina, melhora da atividade das enzimas envolvidas na cascata de sinalização intracelular da insulina, aumento do transporte da proteína GLUT-4 até a membrana plasmática e de sua atividade, diminuição do risco de complicações macrovasculares relacionadas ao DM2, como infarto agudo do miocárdio e acidente vascular encefálico, diminuição da lipólise e dos ácidos graxos livres circulantes, diminuição dos níveis séricos de triglicérides, diminuição da glicogenólise e da gliconeogênese hepáticas, diminuição do ganho de peso corporal e aumento da captação de glicose estimulada pela insulina nos tecidos periféricos, como músculo esquelético e tecido adiposo (10-13).
Os objetivos deste estudo foram produzir modelo experimental para o estudo da SM e analisar os efeitos da metformina sobre a pressão arterial de cauda, o peso corporal, o metabolismo glicídico e o conteúdo de gordura visceral desse modelo experimental.

\section{MÉTODO}

Foram estudados ratos da cepa SHR (Spontaneously Hypertensive Rats - catalogados no Índex Internacional de Animais de Laboratório) fornecidos pelo Centro de Desenvolvimento de Modelos Experimentais para a Medicina (Cedeme) da Universidade Federal de São Paulo (Unifesp), mantidos no Biotério da Disciplina de Nefrologia da mesma instituição em gaiolas coletivas contendo, no máximo, seis animais em cada, permanecendo durante todo o estudo em condições ideais de temperatura $\left(25^{\circ} \mathrm{C}\right)$, umidade ambiental $(55 \%)$ e ciclo luz/escuro com alternância de 12 horas, com acesso livre à água e ração-padrão $\left(\right.$ Purina $\left.^{\circledR}\right)$. O protocolo do estudo foi revisado e aprovado pelo Comitê de Ética em Pesquisa da Unifesp - Escola Paulista de Medicina (EPM) $\left(\mathrm{CEP}^{\circ}{ }^{\circ} 0463 / 2006\right)$.

Inicialmente, os animais foram divididos em dois grupos: SHR e SHR-MSG. O grupo SHR-MSG recebeu injeção subcutânea de $2 \mathrm{mg} / \mathrm{kg}$ de glutamato monossódico (MSG - Sigma, St. Louis, MO, Estados Unidos) em dias alternados, do $2^{\circ}$ até o $11^{\circ}$ dia de vida. Os animais do grupo $\mathrm{SHR}$ receberam o mesmo volume de salina. A partir da $12^{\text {a }}$ semana de vida, as pressões arteriais de cauda (método oscilométrico) e o peso corporal basais foram aferidos em ambos os grupos, e metade dos animais de cada grupo passou a receber, por gavagem, $500 \mathrm{mg} / \mathrm{kg} /$ dia de metformina (cloridrato de metformina, Merck Genéricos), durante mais 12 semanas consecutivas, constituindo, dessa forma, os grupos SHR-Metformina e SHR-MSG-Metformina (SHR-Met e SHR-MSG-Met).

Durante as 12 semanas de tratamento/acompanhamento, a pressão arterial de cauda e o peso corporal foram medidos duas vezes por semana, sendo o valor médio das duas medidas utilizado como representativo daquela semana.

\section{Teste de tolerância à glicose oral}

Após o término do período de tratamento/acompanhamento, todos os animais foram submetidos ao teste de tolerância à glicose oral (TTGO). Vinte e quatro 
horas antes, os animais foram anestesiados com cetamina $(100 \mathrm{mg} / \mathrm{kg})$ e xilasina $(10 \mathrm{mg} / \mathrm{kg})$ por via intraperitoneal e posicionados em decúbito dorsal. Por meio de incisão oblíqua na região inguinal, um cateter de polietileno do tipo PE-10 (Intramedic, Clay Adams, New Jersey, Estados Unidos) foi implantado na artéria femoral. Este cateter estava conectado a um cateter PE50 (CPL, São Paulo, Brasil) de $20 \mathrm{~cm}$ de comprimento. Esses cateteres eram previamente preenchidos com solução salina heparinizada $(10 \mathrm{U} / \mathrm{mL})$. O mesmo cateter PE50 foi passado, com a ajuda de um trocáter, por via subcutânea pelo dorso do animal até a região cervical posterior, onde foi exteriorizado e fixado. Terminado o efeito da anestesia, os animais eram colocados em gaiolas individuais e iniciava-se o jejum para sólidos de 12 horas antes do TTGO.

O primeiro passo do TTGO consistiu na coleta de sangue para determinação da glicemia e insulinemia de jejum. Por meio do cateter arterial, coletou-se 1 gota de sangue para determinação da glicemia e $1 \mathrm{~mL}$ de sangue total em tubo Eppendorf para determinação da insulinemia. Depois de realizada a coleta basal, os animais receberam, por gavagem, $1,7 \mathrm{~g} / \mathrm{kg}$ de glicose anidra diluída em água destilada (14). Novas coletas de sangue foram realizadas $15,30,60,90$ e 120 minutos após administração de glicose.

A glicemia foi determinada utilizando-se um glicosímetro (Optium - Abbott MediSense) e tiras reagentes apropriadas (Optium Point-of-Care-Abbott MediSense). Para a determinação da insulinemia, o volume de sangue coletado nos tubos Eppendorfs foi centrifugado (Eppendorf Centrifuge 5403) à velocidade de $10.000 \mathrm{rpm}$ por $10 \mathrm{mi}-$ nutos a $4^{\circ} \mathrm{C}$. O plasma foi separado, colocado em novos tubos Eppendorfs e armazenado à temperatura de $-70^{\circ} \mathrm{C}$ em freezer (Revco Scientific Inc., Asheville, NC, Estados Unidos). O método empregado foi o de radioimunoensaio, utilizando-se o kit de reagentes Coat-A-Count.

Por fim, os valores de glicemia e insulinemia obtidos no TTGO permitiram o cálculo das áreas sob as curvas de glicose e insulina (ASCG e ASCI) pela regra trapezoidal (15) e, com estes dois dados, foi feito o cálculo do índice de sensibilidade à insulina (ISI), pela fórmula modificada de Sluiter e cols. (16):

$\mathrm{ISI}=10.000 /$ ASCG $\times$ ASCI

Terminado o teste, os animais foram sacrificados por excesso de anestésico para retirada e pesagem da gordura periepidídimo, como representativa da gordura visceral (17). Determinou-se a gordura epididimal relativa expressa por $100 \mathrm{~g}$ de peso corporal.
Para a análise dos resultados, foram utilizados testes paramétricos e testes não paramétricos, levando-se em consideração a natureza das variáveis estudadas e a variabilidade das medidas obtidas. Foram aplicados os seguintes testes: a) análise de variância de medidas repetidas (paramétricas): para comparar as variações temporais da pressão arterial de cauda e do peso corporal. Quando observadas diferenças estatisticamente significativas, foi realizado o teste de comparações múltiplas de Bonferroni; b) análise de variância entre os diferentes grupos (paramétrica): para comparar os valores absoluto e relativo do peso da gordura epididimal. Quando observadas diferenças estatisticamente significativas, foi realizado o teste de comparações múltiplas de Bonferroni; c) análise de variância por postos de Kruskal-Wallis (ANOVA on Ranks - medidas não paramétricas): foi utilizada para comparar os valores do TTGO, ou seja, as áreas sob as curvas de glicose e insulina e o índice de sensibilidade à insulina. Quando existiam diferenças significativas entre os valores comparados, era aplicado o teste de comparações múltiplas de Dunn. Em todos os testes fixou-se o nível de significância em $5 \%$ (p $<0,05)$ para rejeição da hipótese de nulidade.

\section{RESULTADOS}

Os resultados estão apresentados como médias aritméticas dos valores individuais de cada grupo, acompanhadas do erro-padrão da média (EPM), como medida de dispersão (Tabela 1).

\section{Pressão arterial de cauda e peso corporal}

Quando se compara a variação percentual das pressões arteriais de cauda (Figura 1), verifica-se que a produção de obesidade neuroendócrina pela administração de glutamato monossódico a ratos SHR não alterou esse parâmetro, uma vez que a variação observada nos animais do grupo SHR-MSG e do grupo-controle (SHR) não revelou diferença estatisticamente significativa. Já o tratamento com metformina reduziu significativamente a pressão arterial de cauda nos dois grupos tratados (SHR-Met e SHR-MSG-Met).

Quando se realiza a mesma análise para o peso corporal (Figura 2), não se observam alterações significativas com a administração neonatal de glutamato monossódico, embora o ganho de peso corporal tenha sido um pouco menor neste grupo. Porém, o tratamento com metformina produziu diminuição significativa do peso corporal nos animais SHR-Met, mas não nos animais SHR-MSG-Met. 
Tabela 1. Valores médios \pm EPM da pressão arterial de cauda no período basal e na $12^{\mathrm{a}}$ semana do estudo $(\mathrm{mmHg})$ e variação percentual ( $\left.\triangle P A C \%\right)$. Valores médioS \pm EPM do peso corporal basal e da $12^{\mathrm{a}}$ semana $(\mathrm{g})$ e variação percentual ( $\triangle \mathrm{PC} \%$ ). Valores médios \pm EPM das áreas sob as curvas de glicose (ASCG, $\left.\mathrm{mg} / \mathrm{dL}\right)$ e insulina (ASCl, $\left.\mu \mathrm{U} / \mathrm{mL}\right)$, índice de sensibilidade à insulina (ISI, $\mathrm{mg}^{-1}$. $\left.\mu \mathrm{U}-1 . \mathrm{ml}^{-1}\right)$ e gordura epididimal relativa (GER, g/100 g)

\begin{tabular}{|c|c|c|c|c|}
\hline Parâmetro & SHR & SHR-MSG & SHR-Met & SHR-MSG-Met \\
\hline n & 10 & 5 & 8 & 10 \\
\hline PAC basal & $174 \pm 2,83$ & $180,4 \pm 8,69$ & $174,5 \pm 5,09$ & $156,7 \pm 2,75$ \\
\hline PAC $12^{\mathrm{a}}$ sem. & $197,3 \pm 4,12$ & $202 \pm 8,06$ & $160 \pm 2,43$ & $146,8 \pm 2,85$ \\
\hline$\triangle P A C$ & $+13,37 \pm 3,11$ & $+11,91 \pm 3,81$ & $-7,81 \pm 2,58^{\star}$ & $-3,63 \pm 2,61^{\sharp}$ \\
\hline PC basal & $256,5 \pm 4,53$ & $246,4 \pm 14,47$ & $252,25 \pm 3,95$ & $212,5 \pm 4,26$ \\
\hline PC $12^{\mathrm{a}}$ sem. & $323,2 \pm 4,84$ & $290,2 \pm 17,38$ & $276,87 \pm 5,2$ & $254,8 \pm 6,58$ \\
\hline$\triangle P C$ & $+26,11 \pm 1,21$ & $+17,75 \pm 1,32$ & $+9,87 \pm 2,12^{*}$ & $+19,89 \pm 1,82$ \\
\hline ASCG & $237,54 \pm 15,81$ & $261,15 \pm 5,28^{*}$ & $148,66 \pm 11,03^{*}$ & $198,96 \pm 15,55^{*}$ \\
\hline $\mathrm{ASCl}$ & $2,44 \pm 0,12$ & $21,68 \pm 1,71^{\star}$ & $2,51 \pm 0,17$ & $1,85 \pm 0,06^{\#}$ \\
\hline$|S|$ & $18,59 \pm 1,45$ & $1,66 \pm 0,14^{*}$ & $29,04 \pm 3,23^{*}$ & $29,19 \pm 2,63^{\sharp}$ \\
\hline GER & $1,17 \pm 0,03$ & $1,71 \pm 0,08^{\star}$ & $0,90 \pm 0,06^{*}$ & $0,92 \pm 0,04^{\#}$ \\
\hline
\end{tabular}

${ }^{*} p<0,05$ versus SHR; $\# p<0,05$ versus SHR-MSG.

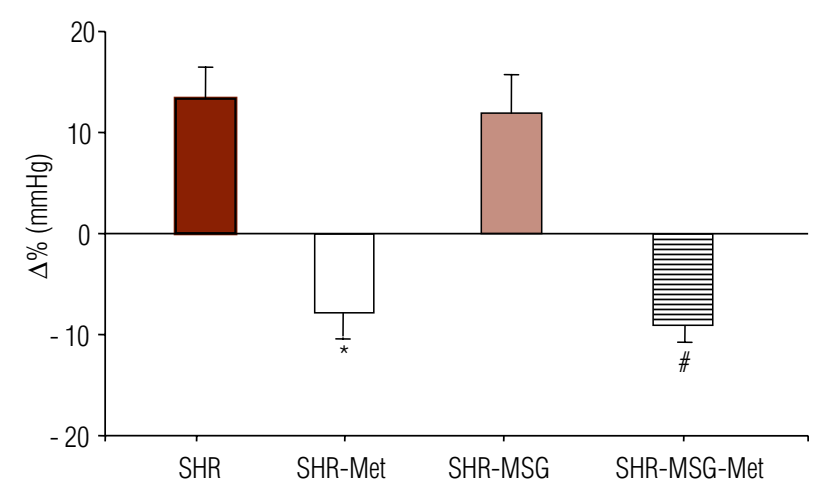

${ }^{*} p<0,05$ versus SHR; $\# p<0,05$ versus SHR-MSG.

Figura 1. Valores médios da variação percentual da pressão arterial de cauda \pm EPM, dos grupos SHR, SHR-Metformina, SHR-MSG e SHR-MSG-Metformina nas 12 semanas de estudo.

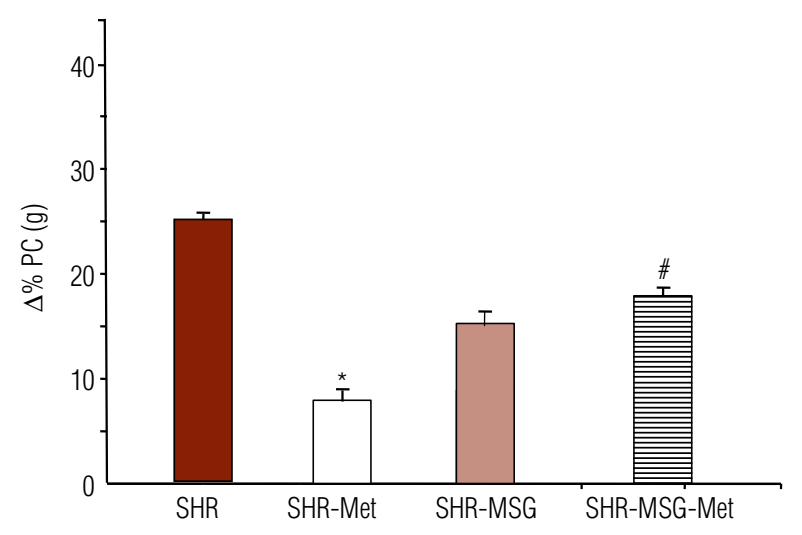

* $p<0,05$ versus $S H R ; \# p<0,05$ versus SHR-MSG.

Figura 2. Valores médios da variação percentual do peso corporal \pm EPM, dos grupos SHR, SHR-Metformina, SHR-MSG e SHR-MSG-Metformina nas 12 semanas de estudo.

\section{Parâmetros metabólicos}

\section{Áreas sob as curvas de glicose e insulina}

Nota-se que a administração neonatal de MSG aos ratos SHR leva ao aumento significativo da área sob a curva de glicose e que o tratamento com metformina diminuiu significativamente este parâmetro, tanto no grupo SHRMet quanto no grupo SHR-MSG-Met (Figura 3).

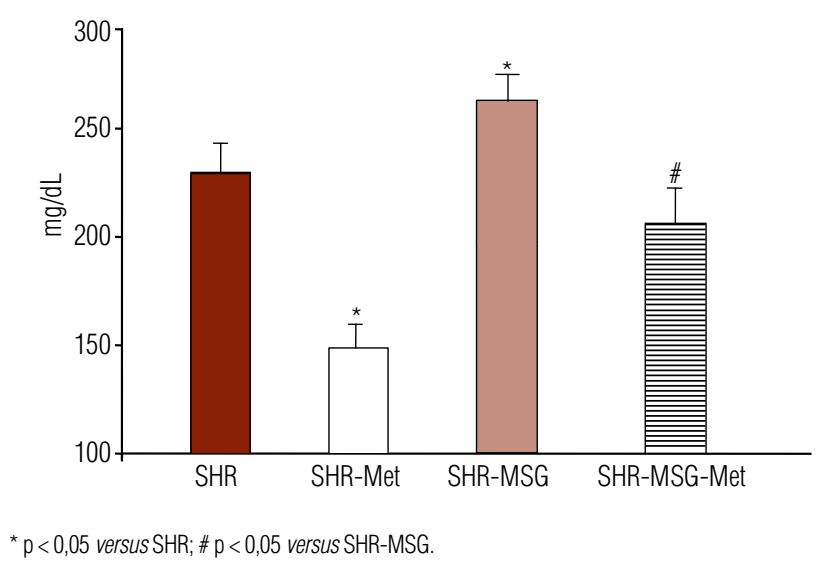

Figura 3. Valores médios da área sob a curva de glicose, em $\mathrm{mg} / \mathrm{dL} \pm$ EPM, dos grupos SHR, SHR-Metformina, SHR-MSG e SHR-MSG-Metformina durante 0 teste de tolerância à glicose oral.

Verifica-se também aumento significativo na área sob a curva de insulina entre os animais SHR que receberam MSG no período neonatal e foram tratados com metformina. No grupo SHR-MSG-Met, o tratamento foi capaz de diminuir a insulinemia durante o TTGO (Figura 4). 


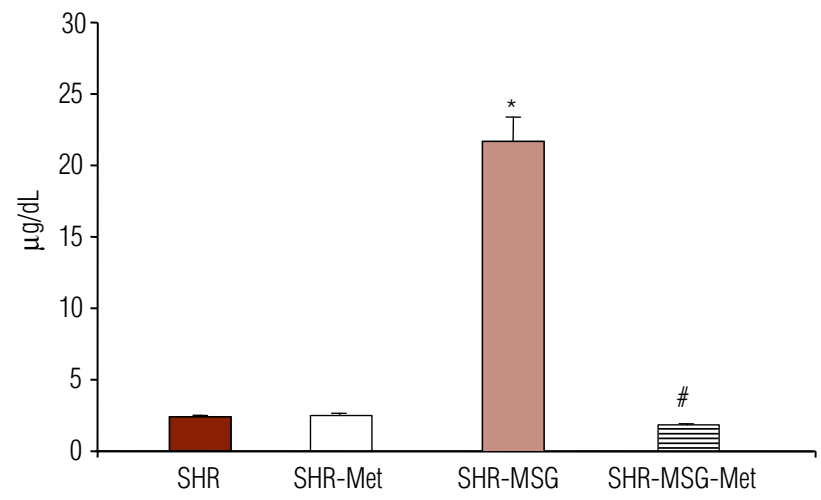

${ }^{*} \mathrm{p}<0,05$ versus SHR; $\# \mathrm{p}<0,05$ versus SHR-MSG.

Figura 4. Valores médios da área sob a curva de insulina, em $\mu \mathrm{U} / \mathrm{mL} \pm E P M$, dos grupos SHR, SHR-Metformina, SHR-MSG e SHR-MSG-Metformina durante 0 teste de tolerância à glicose oral.

\section{Îndice de sensibilidade à insulina}

O tratamento neonatal com MSG determinou diminuição do índice de sensibilidade à insulina. Ambos os grupos tratados com metformina (SHR-Met e SHRMSG-Met) apresentaram aumento significativo deste parâmetro quando comparados aos seus respectivos controles (SHR e SHR-MSG). Dessa forma, vê-se melhora da sensibilidade à insulina após tratamento com metformina (Figura 5).

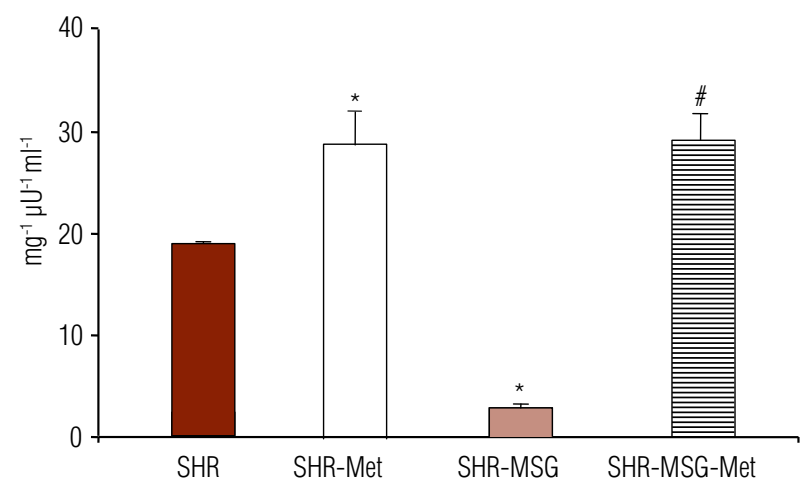

${ }^{*} p<0,05$ versus SHR; $\# p<0,05$ versus SHR-MSG.

Figura 5. Valores médios do índice de sensibilidade à insulina, $\mathrm{em} \mathrm{mg}^{-1} \cdot \mathrm{\mu U}^{-1} \cdot \mathrm{ml}^{-1} \pm$ EPM, dos grupos SHR, SHR-Metformina, SHR-MSG e SHR-MSG-Metformina durante 0 teste de tolerância à glicose oral.

\section{Gordura epididimal}

A administração neonatal de MSG também levou ao aumento significativo na deposição de gordura visceral. Todos os animais tratados com metformina (SHR-Met e SHR-MSG-Met) apresentaram diminuição significativa do conteúdo de gordura epididimal (Figura 6).

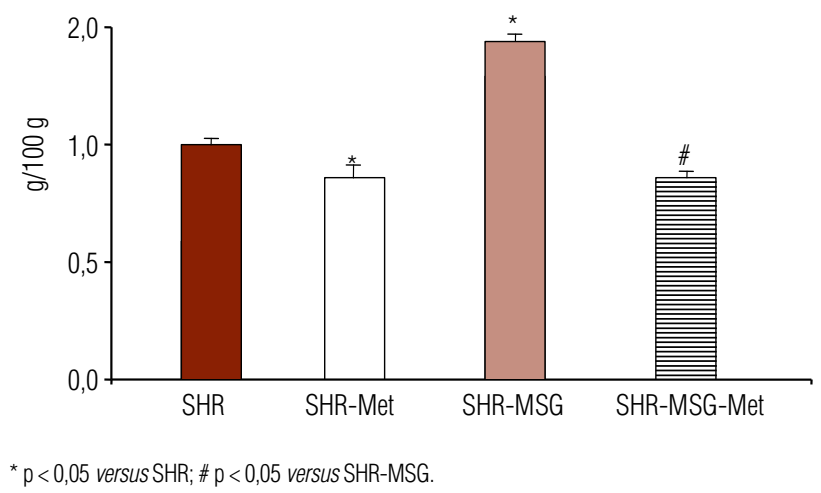

Figura 6. Gordura epididimal relativa: valores médios em $g / 100 \mathrm{~g} \pm$ EPM, dos grupos SHR, SHR-Metformina, SHR-MSG e SHR-MSG-Metformina.

\section{DISCUSSÃO}

Os resultados deste estudo mostram dois aspectos relevantes. O primeiro deles é o desenvolvimento de um modelo experimental que associa a obesidade neuroendócrina com a hipertensão arterial neurogênica dos ratos espontaneamente hipertensos machos. O modelo experimental obtido tem hipertensão arterial, é mais resistente à ação periférica da insulina, tem aumento do conteúdo de gordura visceral e mimetiza, em parte, situação da SM. Outro achado importante é que o tratamento com metformina reduz o conteúdo de gordura visceral e, com isso, melhora a sensibilidade à insulina, além de promover diminuição da pressão arterial de cauda.

O rato SHR é um modelo de hipertensão arterial genética, que se assemelha à hipertensão essencial humana, sendo, assim, amplamente utilizado em estudos experimentais. A hipertensão arterial deste modelo experimental tem como etiologia primária a hiperatividade adrenérgica $(18,19)$. Esse animal também é resistente à ação da insulina, seja pela própria hiperatividade simpática, seja por apresentar defeito na captação de glicose, esta mediada pela mutação do alelo CD36. Apesar de todas as alterações metabólicas, os ratos SHR não são obesos e seu conteúdo de gordura visceral assemelha-se a ratos normotensos Wistar (20-22).

A administração de MSG aos ratos do grupo SHR determinou menor ganho de peso corporal dos animais, não obstante o aumento do conteúdo de gordura visceral. Sabe-se que a administração de MSG pode se acompanhar de diminuição do peso corporal, pois provavelmente a ação neurotóxica dessa droga determine menor secreção de hormônio do crescimento, o que determina animais com diminuição do comprimento nasocaudal. Apesar do menor peso corporal, a administração de MSG determinou importante aumento da 
gordura visceral, o que intensificou a resistência à insulina, sem, no entanto, promover elevação adicional da pressão arterial de cauda. Essa observação era inesperada, pois na gênese da hipertensão arterial secundária a obesidade apresenta os mesmos fatores presentes nos ratos SHR-MSG, como aumento da gordura visceral, hiperatividade simpática, hipercortisolismo (mediado pela administração de MSG), aumento da leptina, diminuição da adiponectina e aumento de adipocinas (23-27). No entanto, um estudo realizado por Iwase e cols. (28) mostra dados que corroboram pelo menos em parte com o presente trabalho. Esses pesquisadores também administraram MSG no período neonatal a ratos SHR e observaram até redução da pressão arterial de cauda após 14 semanas de vida, que se manteve, porém, em patamares de hipertensão arterial. A justificativa dos autores fundamentou-se no fato de que o MSG leva à interrupção do eixo hipotálamo-hipófise-adrenal, cujo funcionamento deve estar intacto para o desenvolvimento da hipertensão arterial dos ratos SHR. Assim tomando em conjunto o presente trabalho e o de Iwase e cols., pode-se afirmar que embora a administração neonatal de MSG ative mecanismos potencialmente pressores, não ocorre sinergismo com aqueles que são ativados na hipertensão arterial experimental espontânea.

Porém, não obstante esses fatores não determinaram elevação da pressão arterial, contribuíram para elevar as áreas sobre as curvas de glicose e insulina, isto é, diminuíram a captação periférica de glicose e insulina de ratos SHR-MSG. A resultante desses dois fatores mostra resistência à insulina aumentada em relação aos ratos SHR e, dessa forma, produziu-se modelo experimental útil para o estudo da SM.

A importância da metformina para o tratamento de pacientes diabéticos foi ressaltada no estudo UKPDS (United Kingdom Prospective Diabetes Study), no qual aqueles que fizeram uso dessa droga apresentaram menor número de eventos cardiovasculares, apesar do controle metabólico semelhante àqueles que não usaram a metformina (29).

A administração de metformina aos animais SHR produziu diminuição da pressão arterial, da gordura visceral relativa e melhora da resistência à insulina. Com exceção da diminuição da pressão arterial, o mesmo ocorreu com os ratos SHR-MSG, embora a redução da pressão arterial não tenha sido significativa.

Os efeitos benéficos da metformina neste modelo experimental podem ser atribuídos à diminuição do conteúdo de gordura visceral verificado nestes animais, pois já é bem sabido que entre seus efeitos farmacológicos se verificam diminuição da lipogênese, diminuição da oxidação de ácidos graxos, além de melhora na captação muscular e hepática de glicose. Também é conhecido que a metformina diminui a lipotoxicidade e melhora a sinalização da insulina (30-39). Como já mencionado, a diminuição da gordura visceral contribui com a diminuição da atividade simpática, a diminuição da atividade do sistema renina-angiotensina-aldosterona e o aumento da liberação de óxido nítrico.

Conclui-se que a injeção neonatal de MSG induz obesidade visceral e agrava os distúrbios do metabolismo glicídico nos animais da cepa SHR, mas não altera os níveis pressóricos. O tratamento com a metformina melhora o metabolismo glicídico e reduz a pressão arterial nesse modelo experimental de SM, além de reduzir a deposição de gordura visceral. Essas observações apontam para o importante papel da resistência hepática à insulina nos distúrbios metabólicos nesse modelo e evidenciam os efeitos cardiovasculares benéficos da melhora na sensibilidade à insulina.

Agradecimentos: este trabalho foi realizado com o apoio da Fundação Oswaldo Ramos.

Declaração: os autores declaram não haver conflitos de interesse científico neste estudo.

\section{REFERÊNCIAS}

1. Miranda PJ, DeFronzo RA, Califf RM, Guyton JR. Metabolic syndrome: definition, pathophysiology, and mechanisms. Am Heart J. 2005;149:33-45.

2. Pladevall M, Singal B, Williams LK, Brotons $C$, Guyer H, Sadurni J, et al. A single factor underlies the metabolic syndrome. Diabetes Care. 2006;29:113-22.

3. Alberti KGMM, Zimmet P, Shaw J. Metabolic syndrome - A new world-wide definition. A Consensus Statement from the International Diabetes Federation. Diabet Med. 2006;23:469-80.

4. Astrup A. The metabolic syndrome. $N$ Engl J Med. 2006:354:2735-6.

5. Eckel RH, Grundy SM, Zimmet PZ. The metabolic syndrome. Lancet. 2005;365:1415-28.

6. Bahia L, Aguiar LGK, Villela NR, Bottino D, Bouskela E. O endotélio na síndrome metabólica. Arq Bras Endocrinol Metab. 2006;50:291-303.

7. Siqueira AFA, Abdalla DSP, Ferreira SRG. LDL: da síndrome metabólica à instabilização da placa aterosclerótica. Arq Bras Endocrinol Metab. 2006;50:334-43.

8. McGarry JD, Dobbins RL. Fatty acids, lipotoxicity and insulin secretion. Diabetologia. 1999;42:128-38.

9. Cesaretti MLR, Kohlmann O. Modelos experimentais de resistência à insulina e obesidade: lições aprendidas. Arq Bras Endocrinol Metab. 2006;50:190-7.

10. I Diretriz Brasileira de Diagnóstico e Tratamento da Síndrome Metabólica. Arq Bras Cardiol. 2005;84(Suppl 1):1-28. 
11. Goodarzi MO, Bryer-Ash M. Metformin revisited: re-evaluation of its properties and role in the pharmacopoeia of modern antidiabetic agents. Diabetes Obes Metab. 2005;7:654-65.

12. Holland W, Morrison T, Chang Y, Wiernsperger N, Stith BJ. Metformin (Glucophage) inhibits tyrosine phosphatase activity to stimulate the insulin receptor tyrosine kinase. Biochem Pharmacol. 2004;67:2081-91.

13. Bailey CJ.Treating insulin resistance in type 2 diabetes with metformin and thiazolidinediones. Diabetes Obes Metab. 2005;7:675-91.

14. Lyon AW, Larsen ET, Edwards AL. The impact of new guidelines for glucose tolerance testing on clinical practice and laboratory services. CMAJ. 2004;171:1067-9.

15. Monaco JH, Anderson RL. Tai's formula is the trapezoidal rule. Diabetes Care. 1994;17:1224-5.

16. Tai MM. A mathematical model for the determination of total area under glucose tolerance and other metabolic curves. Diabetes Care. 1994;17:152-4.

17. Gouveia LMFB, Kettelhut IC, Foss MC. Abnormalities of glucose metabolism in spontaneously hypertensive rats. Braz J Med Biol Res. 2000;33:1357-62.

18. Hughes JM, Bund SJ. Arterial myogenic properties of the spontaneously hypertensive rat. Exp Physiol. 2002;87:527-34.

19. Kramer JM, Waldrop TG. Spontaneously hypertensive rats exhibit altered cardiovascular and neuronal responses to muscle contraction. Exp Physiol. 2001;86:717-24.

20. Glazier AM, Scott J, Aitman TJ. Molecular basis of the Cd36 chromosomal deletion underlying SHR defects in insulin action and fatty acid metabolism. Mamm Genome. 2002;13:108-13.

21. Pravenec M, Landa V, Zidek V, Musilova A, Kazdová L, Qi N, et al. Transgenic expression of $\mathrm{Cd} 36$ in the spontaneously hypertensive rats is associated with amelioration of metabolic disturbances but has no effect on hypertension. Physiol Res. 2003;52:681-8.

22. Hajri T, Ibrahimi A, Coburn CT, Knapp FF, Kurtz T, Pravanec M, et al. Defective fatty acid uptake in the spontaneously hypertensive rats is a primary determinant of altered glucose metabolism, hyperinsulinemia, and myocardial hypertrofy. J Biol Chem. 2001;276(26):23661-6.

23. Marmo MR, Nunes MT, Volpato CT, Kettelhut IC, Heel NS, Lima $\mathrm{FB}$, et al. Reduced growth hormones mRNA levels in 28-d-old MSG rats impair protein and lipid metabolism. European J Physiol. 1994;427:R29.

24. Nascimento Curi CMO, Marmo MR, Egami M, Ribeiro AB, Andrade IS, Dolnikoff MS. Effect of monosodium glutamate treatment during neonatal development on lipogenesis rate lipoprotein lipase activity in adult rats. Biochem Int. 1991;24:927-35.

25. Duloo AG, Young JB. Effects of monosodium glutamate and gold thioglucose on dietary regulation of sympathetic nervous system activity in rodents. Metabolism. 1991;40:113-21.

26. Sanabria ERG, Pereira MFS, Dolnikoff MS, Andrade IS, Ferreira AT, Cavalheiro EA, et al. Deficit in hippocampal long-term poten- tiation in monosodium glutamate-treated rats. Brain Res Bull. 2002;59:47-51.

27. Hirata $A E$, Alvarez-Rojas F, Carvalheira JBC, Carvalho CRO, Dolnikoff MS, Saad MJA. Modulation of IR/PTP1B interaction and downstream signaling in insulin sensitive tissues of MSG-rats. Life Sci. 2003;73:1369-81.

28. Iwase M, Ichikawa K, Tashiro K, lino K, Shinohara N, Ibayashi S, et al. Effects of monosodium glutamate-induced obesity in spontaneously hypertensive rats vs. Wistar Kyoto rats: serum leptin and blood flow to brown adipose tissue. Hypertens Res. 2000;23:503-10.

29. UK Prospective Diabetes Study. Effect of intensive blood-glucose control with metformin on complications in overweight patients with type 2 diabetes (UKPDS 34). Lancet. 1998;352:854-65.

30. Luna B, Feinglos MN. Oral agents in the management of type 2 diabetes mellitus. Am Fam Physician. 2001;63:1747-56, 1759-80.

31. Freemark $M$, Bursey $D$. The effects of metformin on body mass index and glucose tolerance in obese adolescents with fasting hyperinsulinemia and a family history of type 2 diabetes. Pediatrics. 2001;107:55-62.

32. Kefas BA, Cai Y, Kerckhofs K, Ling Z, Martens G, Heimberg H, Pipeleers $D$, Van de Casteele M. Metformin-induced stimulation of AMP-activated protein kinase in beta-cells impairs their glucose responsiveness and can lead to apoptosis. Biochem Pharmacol. 2004;68:409-16

33. Strowig SM, Raskin P. Combination therapy using metformin or thiazolidinediones and insulin in the treatment of diabetes mellitus. Diabetes Obes Metab. 2005;7:633-41.

34. Luna V, Casauban L, Sajan MP, Gomez-Daspet J, Powe JL, Miura $A$, et al. Metformin improves atypical protein kinase $C$ activation by insulin and phosphatidylinositol-3,4,5-(PO4)3 in muscle of diabetic subjects. Diabetologia. 2006;49:375-82.

35. Mahrouf M, Ouslimani N, Peynet J, Djelidi R, Couturier M, Therond $P$, et al. Metformin reduces angiotensin-mediated intracellular production of reactive oxygen species in endothelial cells through the inhibition of protein kinase C. Biochem Pharmacol. 2006;72:176-83.

36. Natali A, Ferrannini E. Effects of metformin and thiazolidinediones on suppression of hepatic glucose production and stimulation of glucose uptake in type 2 diabetes: a systematic review. Diabetologia. 2006;49:434-41.

37. Kumar N, Dey CS. Metformin enhances insulin signalling in insulin dependent and independent pathways in insulin resistant muscle cells. Br J Pharmacol. 2002;137:329-36.

38. Detaille $D$, Wiernsperger N, Devos P. Metformin interaction with insulin-regulated glucose uptake, using the Xenopus laevis oocyte model expressing the mammalian transporter GLUT4. Eur J Pharmacol. 1999;377:127-36.

39. Lupi R, Del Guerra S, Tellini C, Giannarelli R, Coppelli A, Lorenzetti $M$, et al. The biguanide compound metformin prevents desensitization of human pancreatic islets induced by high glucose. Eur $\mathrm{J}$ Pharmacol. 1999;364:205-9. 